\title{
La donna fantasma: immagini del petrarchismo filmico
}

\author{
Gino Frezza
}

Università di Salerno

\section{Abstract}

Anche se la forza e la vastità delle influenze petrarchesche nel cinema debbono talvolta essere cercate e trovate con la pazienza certosina dell'entomologo o dello studioso di metafore, Gino Frezza rintraccia significative e ineludibili presenze delle figure poetiche petrarchesche nella tradizione filmica del cinema classico e in quello moderno e contemporaneo. Il rapporto fra l'opera di Petrarca e il cinema si rivela, seppure in filigrana, nella cruciale, enigmatica, valenza di un crocevia culturale non secondario, ricorrente, denso di sorprese.

Prima Frezza ripercorre come Petrarca, nelle rime del Canzoniere, trama la difficile, complessa, interfaccia fra rima e immagine, dimensione della scrittura e configurazione visiva, e come, per tale interfaccia, si imponga la figura della Donna nei termini di una immagine ad alto coefficiente fantasmatico. Poi l'autore illustra la permanenza dei temi poetici del Petrarca in un ambito di produzioni filmiche appartenenti ad autori ed epoche del Novecento che, con l'opera del poeta italiano, non hanno rapporti espliciti né affiliazioni o vicinanze metaforiche. Ecco dunque la «donna fantasma» petrarchesca trasformarsi nelle fisionomie filmiche della donna-quadro e della donna-specchio, della donna-statua, della donna-ricordo e, infine, della donna-idea.

L'analisi delle influenze petrarchesche nel cinema si conclude ascrivendo al poeta italiano trecentesco l'origine più lucidamente consapevole di una teoria delle lacrime, che non a caso e molto spesso coprono d'improvviso gli occhi degli spettatori di cinema. Le lacrime spettatoriali — una esperienza insieme fisica e lirica, manifesta e intima - corrispondono alle lagrime petrarchesche, a fronte della vanità terrena dei mortali.

Parole chiave: Mute Lingue, immagine, fantasma, trasparenza, passione/desiderio, visibile/invisibile, lacrime, spettatore.

\section{Abstract}

The strength and magnitude of Petrarch's influence on cinema must sometimes be sought out with the Carthusian patience of the entomologist or like the study of metaphors. Nevertheless, Gino Frezza retraces the significant and unavoidable presence of Petrarchan poetic figures in the film tradition of both classic and modern, contemporary cinema. The connection between the work of Petrarch and the cinema is revealed, though delicately, in the crucial, enigmatic strength of an early, recurrent cultural crossroads, thick with surprise. 
Like Petrarch, Frezza first retraces the rhymes of the Canzoniere, plotting the difficult, complex interface between rhyme and image, the dimensions of writing and visual configuration, and how, for such interface, the figure of the Woman was imposed as a highly ghost representation. The author then illustrates the continuity of Petrarch's poetic themes in a film production environment pertinent to authors and periods from the $10^{\text {th }} \mathrm{Centu-}$ ry which, with the work of the Italian poet, bore no explicit relation nor metaphoric affiliation or similarity. It was here, therefore, that the Petrarchan "ghost woman» was transformed into the cinematic physiognomies of the portrait-woman, mirror-woman, statue-woman, memory-woman and, finally, the idea-woman.

The analysis of Petrarchan influences on cinema is concluded by crediting the $4^{\text {th }}$ Century Italian poet with the most lucidly conscious origin of a theory of tears, which very often, and not unintentionally, covers the eyes of cinema audiences. Spectator tears - an experience both physical and lyrical, open yet intimate- are significant of Petrarchan tears, relating to the earthly pride of mortals.

Key words: Silent Languages, images, ghost, transparency, passion/desire, visible/invisible, tears, spectators.

\section{PARTE PRIMA}

\section{Mute Lingue}

Petrarca è poeta intimamente cinematografico per le modalità con cui trasforma la figura della donna in immagine. È noto come i temi della finestra e del quadro, dello sguardo interiore e della visione fantasmatica ${ }^{1}$ siano essenziali e decisivi nella sua produzione poetica; nel Canzoniere Petrarca pone in gioco modalità e oggetto di uno sguardo che si nutre d'immaginazione. Petrarca pone la lingua e la poesia davanti all'insorgenza delle figure dell' immaginario $^{2}$ rendendo queste ultime una componente fondamentale del senso fornito alle parole e ai segni verbali della poesia. Con Petrarca, in modo sistematico, la lingua e la poesia si dispongono frontalmente alla sfida di restituire l'incombente fisionomia di percezioni visive che, mentre sembrano incise nel dispiegarsi oggettivo dello sguardo, risultano altrettanto inscritte nel malinconico, fantasmatico, ripiegamento di occhi interiori. ${ }^{3}$ Le immagini evocate poeticamente da Petrarca, per quanto spazialmente immobilizzate in quadri o cornici, possiedono un pathos che le rende emotivamente dinamiche, dunque cinematografiche.

1. Marco SAntagata, I frammenti dell'anima. Storia e racconto nel Canzoniere di Petrarca, Bologna: Il Mulino, 1992-2004, p. 216-242.

2. Edgar MORIN, Le cinéma ou l'homme imaginaire, Paris: Minuit, 1956 (tr. it., Il cinema o l'uomo immaginario, Milano: Feltrinelli, 1982).

3. Giorgio AGAMBEN, Stanze. La parola e il fantasma nella cultura occidentale, Torino: Einaudi, 1979 . 


\section{II}

Che la poesia del Petrarca, collegata con spiccata originalità a quella trobadorica e a quella stilnovista, sia marcatamente visiva è un fatto acclarato. ${ }^{4}$ Petrarca suggerisce e stimola visioni e immagini sia tratteggiando, in rima, i contorni e gli elementi dell'ambiente (X, 5-9: ...qui non palazzi, non theatro o loggia, / ma 'n lor vece un abete, un faggio, un pino, / tra l'erba verde e 'l bel monte vicino,/ onde si scende poetando et poggia,/ levan di terra al ciel nostro intellecto...) per cui la scena (qui... non palazzi...ma...) si salda immediatamente all'impulso che eleva il pensiero (levan di terra al ciel nostro intellecto), e quindi l'ambiente configurato dalle rime non ha il segno naturalistico ma l'ideale forma del quadro prospettico del figurativismo umanistico e rinascimentale; sia evocando la parvenza interna, in trasparenza, nell'interiorità dell'anima, del volto di Donna (XI: dall'attacco, 1-2, Lassare il velo o per sole o per ombra,/ Donna, non vi vid'io/... al finale, 12-14, si mi governa il velo, / che per mia morte, et al caldo et al gielo, / de' be' vostr'occhi il dolce lume adombra), che dunque curva in una dimensione intima, rafforzata da verbi (XIII, 5-6: I benedico il loco e 'I tempo et l'oral che sì alto miraron gli occhi miei...; o ancora in XVII, 8: "mentr'io son a mirarvi intento et fiso»; e infine, dopo innumeri ritorni, alla CCCXXV, 39-45: Ma sì com'uom talor che piange, et parte,/ vede cosa, che li occhi e 'l cor alletta, / cosi Colei, per ch'io son in pregione,/ standosi ad un balcone,/ che fu sola a' suoi dì cosa perfecta,/ cominciai a mirar con tal desio,/ che me stesso e 'l mio mal posi in oblio) che indicano il primato di uno sguardo, quasi allucinato ("intento et fiso»), teso a catturare o l'animosa leggiadria (XIII, 12) o lo statuto visivamente emblematico (CCCXXV, 42-43: standosi ad un balcone, / che fu sola a' suoi dì cosa perfecta) dell'immagine fantasmatica inseguita. Tale è lo sforzo intenso profuso a mirar tali immagini, che il poeta si dice non a caso, più volte, lasso e di tale sua marcatura esemplarmente, alla canzone XIX, 9-14, indica: Ch'i non son forte ad aspectar la lucel di questa Donna, et non so fare schermil di luoghi tenebrosi, o d'ore tarde./ Però con gli occhi lacrimosi e 'nfermil mio destino a vederla mi conduce; et so ben ch'i vo dietro a quel che m'arde.

\section{III}

Appurati i percorsi per i quali la poesia di Petrarca determina l'intento di inseguire l'immagine ideale; verificato che la sua poesia trama la difficile complessa interfaccia fra rima e immagine, dimensione della scrittura e configurazione visiva, va notato che per tutto il Canzoniere il grande poeta sonda le soglie attraverso cui la parola, la rima, il suono, le lingue, possono dar conto della pregnanza di una tale complessa immagine. Due poesie paiono esattamente dedite a de-limitare l'ambito proprio per cui verbo, suono, rima, competono in difetto con il senso che l'immagine -interiore, intima, 
ideale, metaforica, vertiginosamente esistenziale e dunque originariamente non letteraria - possiede in sé; il poetare del Petrarca è come girasse attorno a tali limiti, ogni volta cercando i varchi per cui la destinazione del senso possa infrangersi, o meglio rivelarsi. Reciprocamente: verso e immagine; idea-ricordo di Donna o d'Amor o di Madonna o di Lei (quindi di Laura) e operazione poetica (il lavoro stesso del poetare con la lingua), costituiscono i poli attorno a cui Petrarca tenta di superare quel che in modo immanente è di ciascun ambito. Spesso il poeta dispera di cogliere o rendere quel che trascorre fra l'uno e l'altro ma, pur quando lamenta mancanze o difetti delle sue capacità o delle inefficienze strumentali a sua disposizione, egli traccia e segna ponti, collegamenti, usci mediani o intermediali fra rima e immagine dei quali la cultura occidentale europea, legittimamente, non ha potuto né voluto fare a meno. Nella canzone XX, appena dopo aver puntualizzato, con l'animo di chi Vergognando talor chancor si taccia, / Donna, per me vostra bellezza in rima, / ricorro al tempo ch'i vi vidi primal tal che null'altra fia mai che mi piaccia (1-4), Petrarca subito constata che a ciò non sempre corrisponde un risultato confacente, conseguente, o corrispondente al fatto di vincere poeticamente quel chancor si taccia; aggiunge difatti (dopo un «Ma» estremamente significativo dell'impedimento che qui si cerca di sottolineare) che trovo peso non da le mie braccia, I né ovra da polir colla mia lima; / però l'ingegno, che sua forza estima, / ne l'operatïon tutto s'agghiaccia (5-8); il suo straordinario tentativo, insomma, s'infrange davanti a un compito impossibile (9-10: Più volte già per dir le labbra apersi;/ poi rimase la voce in mezzo 'l pecto). Quel ricorrere al tempo ch'i vi vidi prima possiede una intensità tale per cui il poeta naufraga a fronte della pregnanza dell'immagine-idea-ricordo: Ma qual sòn poria mai salir tant'alto?l Più volte incominciai di scriver versi;/ ma la penna et la mano et l'intellectol rimaser vinti nel primier assalto (11-14).

Il tema dell'impossibile restituzione semantica — tramite il verso, la rima, il suono limato come opera dell' intellecto e dell' ingegno - dell'immagine appare estremamente rivelatore. È parallelo al verbo «tacere» che il Petrarca assai significativamente usa, quasi negli stessi termini della XX canzone, nell'esordio della canzone CCCXXV, 1-4: Tacer non posso, et temo non adoprel contrario effecto la mia lingua al core,/ che vorria far honorel a la sua Donna, che dal Ciel n'ascolta. In questa appassionante canzone, l'immagine di Lei, Donna, varia dallo statuto non trattenibile, non vincolato ad alcuna forma di conoscenza terrena (99-100: Sì chiaro à 'l volto di celesti rai,/ che vostra vista in lui non po' fermarse) all'esplicita, finale, terrena indicazione di un più bel corpo (112). Perché una tale oscillazione, che va dall'iniziale Tacer al lungo dipanarsi della scrittura poetica-metaforica del Petrarca, e altresì dall'idea del "volto di celesti rai» a quello del più bel corpo (sia pur nel segno che Morte acerba et rea,/ ... occider non potea, 111-112) della Donna?

5. M. SANTAGATA, op. cit., p. 209-242, riconduce una tale oscillazione, sullo statuto della figura e dell'immagine petrarchesca della donna, al complesso e lungo passaggio dalla Laura «petrosa» a quella «stilnovista». 
Dedita a raffigurare così Colei, per chio son in pregione,/standosi ad un balconel, che fu sola a' suoi dì cosa perfetta (41-43) nel mentre il poeta sancisce la sua pregione o carcere terreno (101), questa lunga canzone sortisce, insieme, sia il risultato di una scrittura d'immagine di Colei/Costei (Quella, per chio ò di morir tal fame,/canzon mia, spense Morte acerba et rea,/che piu bel corpo occider non potea, 110-112) sia una consapevolezza, non contraddittoria bensì quasi apodittica, delle risultanze dell'accanito sforzo del poeta: Tutte lingue son mute, a dir di Lei quel che tu sol ne sai (97-98).

\section{IV}

Più volte il poeta è arso dalle fiamme del desiderio ma, del pari, è slanciato nella tensione malinconica, immaginativa e immaginaria, del tutto ideale e non reale, dell'evocazione di Lei/Colei che costituisce il punto focale, l'attrazione inestinguibile alla quale è destinato il suo permanere in vita. L'ardere del sentimento del poeta, la fiamma che alimenta e trascina il desio, il voler riprendere un contatto tramite l'immagine con una figura risiedente nel passato, in altrove, in sogno o in un simulacro metaforico e mito-poietico, a loro volta sono bilanciati dal curvarsi dello slancio emotivo-patico verso la consapevolezza di un bene che, tanto più perduto e scomparso dal reale e dal presente, tuttavia resta unico aggancio di senso per il durare della vita in attesa della morte. Il pensiero di un ritorno a quel bene supremo che ha la doppia proprietà, insieme, d'esser stato fuggevole (perché tolto dalla Morte) e tuttavia di tornare in maniera incessante (tante volte, nell'idea e nel ricordo) marca per sempre l'esistenza del poeta, con il conforto di un legame stabilito oltre la vita e la morte, sostenendo l'attesa di un ricongiungimento. Nella XXX lirica, Petrarca esprime una precisa teorizzazione concettuale che può essere intesa quale sorta di origine-fondamento da cui, successivamente, parecchi secoli dopo, viene dedotta una intera teoria dell'immagine-simulacro della Donna, in grado di fornire quasi tutti gli elementi sui quali, nel corso del Novecento, viene definita una concezione della diva e del divismo. Una immagine, quella coltivata e proposta dalle rime petrarchesche, la cui qualità, infatti, è di accompagnare e sedurre l'io poetico, immagine imperitura tal ch'i l’ò dinanzi a gli occhi, l ed avrò sempre, ov'io sia, in poggio o 'n riva (5-6). È l'immagine di Giovene donna sotto un verde lauro (1), che il poeta, davanti al passare e trascorrere degli anni (Ma, perché vola il tempo et fuggon gli anni, 13), tuttavia trattiene fermamente, a faro di se stesso e della propria identità più profonda (16-18: seguirò l'ombra di quel dolce lauro,/ per lo più ardente sole et per la neve, / fin che l'ultimo dì chiuda quest'occhi).

L'immagine di tal Donna, dal Petrarca fatta corrispondere - come la tradizione critica sa da tempo per precise ragioni ${ }^{6}$ - al lauro, ovvero alla poe-

6. Cfr. Ugo Foscolo, "Saggio sopra la poesia del Petrarca», in Francesco Petrarca, Canzoniere, Milano: Feltrinelli, 2003. Cfr. Giacomo LeOPARDI, «Note» a Francesco PetrarCA, cit. Cfr. Francesco De SANCTIS, Saggio critico sul Petrarca, Torino: Einaudi, 1983. Cfr. Gianfranco CONTINI, "Preliminari sulla lingua del Petrarca», in Francesco PetrarCa, cit. 1992; cfr. M. SANTAGATA, op. cit., 1992-2004. 
sia, e alla connessione con Amor, resiste anche se il poeta teme di cangiar pria volto et chiomel che con vera pietà mi mostri gli occhi l'idolo mio scolpito in vivo lauro (25-27); e qui, lo studioso del cinema giocoforza constata la precisa rispondenza della teoria dell'immagine di Laura, resistente al tempo e foriera di speranze collocate oltre i cambiamenti della storia e dell'età biologicapersonale, all'individuazione di un "idolo» non a caso scolpito in vivo lauro. Una tale formazione visiva ha i segni decisivi di quella sorta di sovrumanità, di quasi o para-divinità, di stella che fulge in un cielo che è una sommità ideale interiore, che distingue lo statuto simbolico dei divi e delle dive del cinema. ${ }^{7}$ Questa struttura dell'immagine-idolo è frutto di una precisa operazione costruttiva, linguistica-poetica, ciò̀ mediatica, ossia iscritta nel regime espressivo del medium che il poeta modella e perfeziona coi suoi versi (scolpito in vivo lauro). Come le immagini delle stelle del cinema, di divi e dive, quella di Laura mostra vari registri, dall'inaccessibilità o localizzazione extratemporale all'ideale di felicità da essa veicolata, all'intensità desiderante che invade il soggetto nella pastosa e complessa ambiguità di sembianza tanto similmente evocata —cioè quasi senza remore disponibile a palesarsi davanti agli occhi interiori del poeta-, tanto ricorrente e in grado d'informare di sé ogni variazione esistenziale tocchi all'individuo nel corso della vita, quanto sfuggente ovvero rinviante a una esperienza costantemente differita, collocata nell'oltre del simbolo e della metafora.

7. Cfr. Edgar Morin, Les stars, Paris: Editions du Seuil, 1957 (tr. it., I divi, Milano: Mondadori, 1963) e ID., Sociologie, Paris: Fayard, 1984 (tr. it. Sociologia della sociologia, Roma: Edizioni Lavoro, 1987). 


\section{PARTE SECONDA}

\section{Trasparenti Schermi}

È il momento di far emergere come l'eredità petrarchesca —-figurare il tema della Donna e dell'immagine investendo il senso stesso del medium con il quale ci si confronta, la propria posizione culturale e filosofica nel mondo, il quadro delle relazioni fra mondano e oltremondano ecc. - sia penetrata, pur indirettamente tuttavia in modo inequivocabile, nel corpus del cinema novecentesco, modellando una sua forma altamente rappresentativa, dall'elevato valore allegorico.

\section{La donna fantasma}

L'immagine della Donna è tra i principali elementi sui quali viene fondata nel cinema l'attrazione fra schermo e spettatori. La seduzione della bellezza, ${ }^{8}$ dell'innocenza, della malizia, la voluttuosa espressione o la leggiadra, luminosa, apparizione del viso e della figura femminile sullo schermo del cinema, contenuta fra evidenza erotica del corpo e qualità estetica della sagoma, folgora l'attenzione degli spettatori e possiede la qualità di un modello esemplare, al quale si vuol sempre tornare, e dunque tale da muovere le energie del desiderio.?

Lo schermo cinematografico si nutre con significativa - si potrebbe dire necessaria - abbondanza dell'immagine della Donna. Sia che il cinema rappresenti la vita quotidiana di donne qualsiasi, ovvero la straordinaria flagranza della bellezza eterea e archetipica della Donna fatale, passando per il livello medio di figure femminili eterogenee ed eterodosse, tale immagine costituisce uno speciale obbiettivo espressivo-figurativo dinamico, una méta da raggiungere. Mentre colma di senso lo schermo, l'immagine della donna ha la facoltà di condurre ai punti estremi il potere del cinema di esprimere un immaginario esteso, attrattivo sia per il singolo individuo sia per la comunità più vasta dei pubblici internazionali dell'audiovisivo.

Questi confini o punti estremi sono particolarmente in gioco quando il cinema tratta il vasto campo delle immagini della donna fantasma. In una serie di variazioni ossia di differenti immagini relative al tema della Donna e del femminile, lo schermo filmico pratica o vince o risolve la sfida fra visibile e invisibile, esistente e irreale, eterno e corporeo, presente e passato, materiale e immateriale, opaco e trasparente, angelico o demoniaco, solido o, appunto, fantasmatico. Nelle sue diverse tipologie, la donna fantasma (della quale il film più chiaramente paradigmatico è The Uninvited, La casa sulla scogliera, 1944, di Lewis Allen, con Gail Russell e Ray Milland) definisce un ampio range attorno al quale il cinema sperimenta e applica i limiti della sua espressività. Il tema della donna fantasma contiene e articola gli elementi fondamentali con cui il

9. Cfr. E. Morin, Le stars, cit., e ID., Sociologie, cit. 
cinema scandaglia le modalità più significative del suo funzionamento semiotico e della sua efficacia socio-culturale. Quando sullo schermo l'immagine della donna varca la zona d'ombra e viene estratta dall'indefinizione dell'immaginario e dal sincretismo delle sue relazioni, ${ }^{10}$ per essere riconosciuta in una precisa forma audiovisiva con la quale corpi e figure evidenziano una qualità esemplare; quando, altresì, lo schermo consente il passaggio inverso, dall'evidenza della forma erotica e dalla flagranza dell'eterno femminino, o dalla sinfonia dimensionale del primo piano della donna, ${ }^{11}$ in tutti questi casi si mostra lo spettro entro cui funziona un intero registro espressivo e comunicativo, il campo primario occupato da un medium di comunicazione, il cimento che questo intrattiene con il proprio pubblico.

Perché l'immagine della Donna ha il potere di sfidare l'espressività filmica ai suoi punti estremi, di svelarne la fantasmaticità? Essa, tematicamente, investe l'esigenza di riempire o, altrimenti, svuotare di senso lo spazio rappresentativo della visione, la quadratura dello schermo e la sua efficacia spettacolare/comunicativa; di queste sostanze richiama la condizione per cui l'atto medesimo del vedere nel cinema è tanto una pratica percettiva biologica e, insieme, tecnologica (semioticamente organizzata, delimitata nei suoi percorsi generativi $)^{12}$ quanto esperienza dai fondamenti deboli e fugaci, sottoposta a evanescenze incombenti, a dissolvimenti incipienti, a tradimenti o travisamenti sempre possibili. Il cinema manifesta una tale inquietante proprietà fantasmatica del vedere e del percepire stante il movimento incessante ${ }^{13}$ che travolge lo schermo: un movimento che attiene alla medesima natura tecnologica del medium, sottoposto a ininterrotte innovazioni dal periodo delle origini fino alle attuali configurazioni del cinema digitale. ${ }^{14}$ Il movimento e l'assidua trasformazione dello schermo rendono all'esperienza vissuta dagli spettatori in sala molteplici orientamenti che vanno dalla qualità apparentemente docu-

10. Cfr. Edgar Morin, Le cinéma..., cit.

11. Cfr. Jean EPSTEIN, Ėcrits sur le cinéma, Paris: Seghers, 1974 (tr. it. Valentina PASQUALI (a cura di), L'essenza del cinema, Roma: Biblioteca di Bianco\&Nero, 2002). Epstein ne discorre nei termini di fotogenia dell'imponderabile) per sfumare — pian piano o velocemente- i suoi contorni, facendoli rientrare nella zona d'ombra dell'immaginario; oppure quando certi film pongono in dubbio l'idea stessa dell'esistenza dei corpi corrispondenti alle immagini, o ancora viceversa che queste ultime possano a loro volta esser radicate in un ambiente ricostruibile o razionalmente individuabile, cosicché la questione dell'immagine nella sua realtà propria assurge a tema sul quale il cinema spande la forza ermeneutica e dimostrativa delle sue figurazioni mute o sonore (sorta di metacinema paradossalmente rivolto su di sé sul crinale più critico).

12. Cfr. Gino FrezZA, Cinematografo e cinema, Roma: Edizioni Cosmopoli, 1996, e cfr. Francesco CasetTI, Teorie del cinema (1945-1990), Milano: Bompiani, 1993.

13. Cfr. Gilles DeleuZe, Limage-mouvement, Paris, 1984, (tr. it., Limmagine-movimento, Milano: UbuLibri, 1984) e ID., Limage temps, Paris, 1989 (tr. it., Limmagine-tempo, Milano: UbuLibri, 1989).

14. Cfr. Gino Frezza, Cinematografo e cinema, Roma: Edizioni Cosmopoli, 1996; cfr. Alberto ABRUZZESE, Introduzione a René BARJAVEL, Cinema totale. Saggio sulle forme future del cinema, Roma: Editori Riuniti, 2001, p. 7-31; cfr. Lev MANOvicH, Il linguaggio dei nuovi media, Milano: Olivares, 2002. 
mentaristica (pretesamente ontologica) del vedere/ascoltare le immagini sullo schermo ai tratti e alle modalità di una sistematica, shockante, immersione nell'impasto percettivo multidirezionale fra voci, suoni, forme visive e musiche. ${ }^{15}$ Fra l'apparenza documentaristica e la qualità immersiva e fantasmatica dell'immagine filmica, le somiglianze superano le differenze e ogni distinzione spesso s'annulla. Questa particolare fenomenologia, rivelatasi nel grande cinema classico muto e sonoro, si è disposta secondo significative tendenze evolutive, contribuendo al definirsi delle pratiche espressive del cinema moderno almeno dalla prima metà degli anni cinquanta. In Phantom Lady, La donna fantasma, capolavoro dal titolo paradigmatico diretto a Hollywood nel 1944 da Robert Siodmak e tratto da un testo narrativo di William Irish, l'esistenza dell'immagine della donna sullo schermo è messa radicalmente in questione in termini cinematografici. Nel corso del racconto filmico, una signora sparita dalla circolazione, e tanto tenacemente cercata, ad un certo punto emerge come segno energicamente posto in discussione. Si legittima in modo acuto il dubbio che essa sia reale, ovvero una invenzione da parte di un uomo accusato di omicidio. Nella parte finale, con un dispositivo puramente visivo (un veloce movimento della macchina da presa che trascorre fra immagine vuota e poi piena della figura della signora), Siodmak ripropone il nodo dell'apparire e sparire della donna dal campo inquadrato, dunque della sua reale consistenza. Tutto avviene al prezzo di una drammatica consapevolezza vissuta dai protagonisti e dagli spettatori: aver attraversato la terra di nessuno del fantasma e dell'apparenza, del vuoto di senso e del repentino rovesciamento della realtà in incubo, dell'innocenza incolpevole (un uomo ritenuto ingiustamente omicida) in spietata, quasi irreversibile, dannazione (una condanna a morte che pende inesorabile fino all'ultimo istante). Il tema della donna fantasma in questo film palesa un condizionamento e una responsabilità pesante rivestita dall'immagine femminile, quando è evocata con tale convinzione da sfiorare un forte rischio d'insussistenza. Essa mostra come ogni ipotesi ontologica del vedere/ascoltare le immagini sonore possa franare di fronte a sottili crepe o a improvvisi, non programmabili, spostamenti della loro collocazione e del loro significato, dopo i quali resta la sgranatura tipica del fantasma, sostenuta dalla rete dei sensi simbolici-metaforici che connotano il rapporto fra spettatore e schermo.

\section{Donne-Quadri/Specchi}

Fra donne e quadri esiste nel cinema un importante repertorio di occorrenze che rivelano la falsariga o il dominio intermedio, spettrale, della dissolvenza e del trasparente, della sovrimpressione fra corporeo e incorporeo; queste due ultime dimensioni trovano nel quadro un locus estremamente idoneo e adatto a consentire un tramite, un legame, un travaso, una tessitura che il corporeo, 
la solida concretezza della figura umana della donna, intrattiene con la sua dimensione immaginaria. In diversi film, il quadro di donne appeso su pareti di ville antiche o case moderne apre un varco per cui le realtà spaziotemporali esteriormente oggettive scoloriscono in zone altrimenti inesprimibili se non grazie al fatto che il quadro della figura femminile avvera precisi vincoli con mondi che possono rendersi finalmente comprensibili, testimoniati.

Il quadro pone una messe di significati in relazione con vari personaggi di donne e con il loro destino di amanti, di mogli, di anima gemella: figura desiderata, inseguita, adorata, talvolta combattuta e, in qualche caso, anche moralmente negativa. Il quadro ratifica una concreta non-dimostrabilità dei sentimenti (Rebecca, id., 1940, di Alfred Hitchcock); tesse uno straordinario vincolo fra scomparsi e vivi oltre la morte e il tempo oggettivo del mondo naturale (Portrait of Jennie, Ritratto di Jennie, 1948, di William Dieterle); consente lo scorrere di desideri e passioni forse indicibili, tanto intensi da quasi vincere sopra la possibilità dell'omicidio di una donna — ritenuta morta, all'improvviso riappare viva! - della quale un detective s'innamora appunto vedendone il quadro (il classico Laura, Vertigine, 1944, di Otto Preminger, un film persino nel titolo paradigmaticamente "petrarchesco»). Quadri di donne testimoniano o provocano conseguenze tragiche e meandri oscuri della giustizia oltre le colpevoli azioni degli uomini o le inesatte e fallaci ambizioni della legge umana (i capolavori Scarlet Street, Strada Scarlatta, 1946, e The woman in the Window, La donna del ritratto, 1944, di Fritz Lang). Nel gustoso, ironico That lady in ermine (La signora in ermellino, 1948, iniziato da Ernst Lubitsch e, alla sua improvvisa morte, terminato da Otto Preminger), il quadro di una bella donna bionda del passato corrisponde al doppio «cinico» di una donna del presente, meno pronta della prima a sbrigare difficoltà politico-amorose a colpi di finzione criminale.

Anche lo specchio è usato nel cinema quale spazio di transito fra ideale e reale, copia e originale, spirito e materia. Lo specchio raggruma le potenze dell'opacità e della trasparenza. ${ }^{16}$ Mette in gioco una multidimensionale funzione di soglia fra gli universi paralleli del vero e del falso, del doppio e del passaggio fra mondi altrimenti separati. Lo schermo filmico trova con lo specchio una similitudine dai mille risvolti sia ermeneutici sia metaforici. Il tema dello specchio-schermo investe la singolarità del corpo e la sua possibile dematerializzazione: la donna, l'uomo, sono attratti dallo specchio-schermo per ragioni che riguardano principalmente la ricostituzione della loro identità biologica e culturale (ideale) oltre i vincoli della materia e del carnale, la loro tensione a ritrovarsi in uno spazio principalmente affettivo e morale. L'immagine della donna ritratta nel e dal quadro o negli specchi diviene nel cinema elemento che colpisce ossessivamente il suo destinatario maschile o femminile. Il quadro, in particolare, agisce da elemento di tensione per cui donne e uomini psicologicamente s'incatenano all'immagine ch'esso promana, in una sorta di vortice spaziotemporale, fra ieri e oggi, l'antico e il moderno. 
Tramite il nesso fra donna e quadro, lo straordinario Vertigo, La donna che visse due volte, 1958, di Alfred Hitchcock mostra ossessioni quasi paranoiche di un uomo, fissate sul volto e sulle fattezze bionde di una donna che, a sua volta, s'identifica con una figura femminile ritratta su tela in un museo: un assillo insistente e pervicace per il quale - e non solo per effetto di un malvagio intrigola donna amata e ossessivamente inseguita dall'uomo vive «due volte». L'intreccio narrativo di questo capolavoro di Hitchcock, elabora una direzione estremamente concettuale che definisce i personaggi secondo una chiara funzione metaforica. Madeleine, la bionda moglie di un industriale (una affascinante Kim Novak), sembra intimamente presa e psichicamente catturata, in modo misterioso, dal quadro di una donna di metà Ottocento (Carlotta Valdes) morta suicida; l'immagine della donna è da lei rimirata nella galleria del museo della Legion d'Onore di San Francisco, ella è ipnoticamente fissata di fronte al quadro e sembra dimenticare la sua propria personalità e identità, in una possessione che l'estrania e le fa dimenticare ogni cosa; l'ex poliziotto John Ferguson (chiamato dagli amici Scottie, con la fisionomia di James Stewart) a sua volta vede Madeleine che rimira il quadro, ne osserva a distanza la figura, constatando dettagli che riproducono su Madeleine i tratti della donna del quadro (la pettinatura, la collana al collo) e così si scatena un legame che assoggetta l'uomo a pratiche di sguardo che alimentano il desiderio. Questo straordinario film di Hitchcock ricama sui ricorsivi, molteplici rapporti fra sguardi, passioni e affabulazione filmica. ${ }^{17}$ Madeleine è sospesa fra il mondo reale e quello del sogno, Scottie è anche lui trascinato in un labirintico percorso d'innamoramento che lo incatena alle apparenze e alla dedizione per la figura ideale di Madeleine, alle segrete sembianze di una donna «fantasma» (in questi termini, Madeleine è apostrofata da Midge/Barbara Bel Geddes, l'amica da sempre innamorata di Scottie). Infine, l'intreccio narrativo si conclude - tragicamente, entro il vortice passionale di amori controversi, sospesi fra realtà e immaginazione - solo grazie all'incontro fra le tre immagini di donna che attraversano la vita dell'ex poliziotto: la donna vissuta a San Francisco a metà Ottocento e ritratta nel quadro (Carlotta Valdes); Madeleine, che Scottie ritiene si sia gettata dal campanile di una chiesa spagnola a causa della maledetta possessione/identificazione con Carlotta Valdes oltre che per una sua negligenza; la sosia (Judy Barton, una Kim Novak imbrunita) da lui incontrata per strada. Dentro una camera d'albergo, Scottie quasi costringe Judy a vestirsi e truccarsi a somiglianza di Madeleine. In questa sequenza, Hitchcock ha il pregio petrarchesco di far apparire la Judy/Madeleine vera nelle vesti della Madeleine ideale tramite lo sfumato a colori di un flou verde. L'artificio visivo usato da Hitchcock evidenzia in modo inequivocabile la cifra dell'incontro fra immaginario e formale di un viso-corpo-figura. Questa tanto è visibile e si offre allo sguardo mirato dell'ex poliziotto quanto proviene, chiaramente, dal fantastico regno del sogno e del desiderio che — chissà quanto immeritatamente- cerca disperatamente di valicare le barriere fra vita e morte. 


\section{Donne-Statue}

La statua è il simbolo derivante da una pratica artistica tradizionalmente vincolata a rendere le dimensioni e le fattezze del corpo umano (di quello femminile in specie) secondo la falsariga di una equivalenza tridimensionale, dove la solidità della materia, sia pure immobile, possa far trasparire l'anima individuale o l'espressione ieratica di una posa bloccata fra l'azione del corpo biologico in movimento e l'immobilità della materia inorganica dalla quale la statua viene ricavata e scolpita. Nel cinema le statue possiedono numerose valenze concernenti il rapporto fra corpo dell'attore/attrice e fisionomia immaginaria, ideale, della figura del divo e della diva. Esse incarnano l'idea e il quesito relativi alla somiglianza, alla corrispondenza esatta fra corpo statuario e figura viva, fra bellezza estetica della posa e mobilità non reversibile dell'atto che si spende nel presente della vita individuale. La statua in tale falsariga evidenzia un altro nodo emblematico della relazione fra l'immagine presunta e quella reale, fra l'icona virtuale e il corpo che, resosi presente, deve poter accogliere la sfida del modello costituito dalla posa statuaria. Le statue, specie se di donne rappresentate nella loro perfetta bellezza, sono immagini problematiche, a monte delle quali va sciolta la questione della loro aderenza all'idea che la vita può coagularsi e solidificarsi in un simbolo capace di resistere alle usure del tempo, ai cambiamenti del desiderio, alle evoluzioni del gusto, ai processi incessanti dei modelli culturali.

Il rapporto fra corpo della diva e statua in Song of Songs, Il cantico dei cantici, 1933, di Rouben Mamoulian (con Marlene Dietrich) raffigura l'anima dell'amata, un'anima ed un amore poi compromessi a tal punto che il personaggio di Lei demolirà in frantumi la statua che riporta l'immagine di una innocenza calpestata dalla corruzione di un uomo depravato. In questo capolavoro degli anni trenta, la statua del corpo nudo di Marlene Dietrich delinea i tratti angelici e spirituali della giovane ragazza nell'insorgenza del primo amore della sua vita, evoca il tema della grazia che la Statua medesima ricorda a Lei in modo intollerabile, tanto che Marlene la distrugge in pezzi. Tramite il segno della bellezza simbolica della statua, il film inscena il miraggio di un modello che il cinema, nella figura della diva Marlene, tenta di toccare valicando - imprudentemente o oltraggiosamente — la differenza fra l'immagine e il corpo vivo.

In One touch of Venus, Il bacio di Venere, 1948, di William A. Seiter su sceneggiatura di Frank Tashlin e musiche di Kurt Weill, la statua della Venere d'Anatolia si trasforma in corpo vivo (una giovane, irresistibile Ava Gardner) dopo essere stata baciata dal vetrinista di un grande magazzino (Robert Walker). La Venere appare e scompare dai bordi dello schermo — dal fuoricampo- così come rientra in campo dall'immagine riflessa di uno specchio. Sia nel film di Mamoulian che in quello di Seiter/Tashlin, la statua è immagine di una idea; quella dell'anima giovanile della dolce Marlène costretta a contaminarsi con le brutture del mondo; quella dei bordi e dei confini dai quali è consentita la visibilità, fra quadro e fuori campo, fra il terreno e il divino, di una Venere, di una eterna bellezza femminea che, valicando tali confi- 
ni temporaneamente, si concede all'innamoramento di un giovane vetrinista, di un uomo qualsiasi. Statue che corrispondono alla dialettica petrarchesca fra lo statuto ideale della figura dell'amata e la voluttuosa corporeità della donna, capace di far ardere passioni e affetti.

\section{Donna-Ricordo}

Il ricordo nel cinema scatena un altro strato problematico che attiene alla verità e aderenza dell'immagine del presente a ciò che irreversibilmente si situa nel passato. Il ricordo nel cinema mette in questione tutti i passaggi con cui si rende percepibile, afferrabile, consumabile, un legame fra ieri e oggi. Tra le molte fisionomie della memoria depositate nelle tipologie narrative e concettuali del cinema ${ }^{18}$ una, specificamente, richiama il bordo fra ciò che non è visto o non ancora riconosciuto nella sua evidenza figurale e ciò che "vibra» premendo sui contorni dell'inquadratura per essere, finalmente, ammirato nella sua flagranza audiovisiva. È un altro, decisivo spazio della dissolvenza e del trasparente filmico, puntualizzato nel momento dell'insorgenza iniziale della visione e non su quello della sua sparizione, del suo dileguarsi. Molti film sui temi del quadro, dello specchio e della statua riprendono questo meccanismo di trasparenza e dissolvenza dell'immagine femminile, ma un caso inconsueto riveste una specifica importanza ed ha rilievo per la ricerca di immagini dal sapore intensamente "petrarchesco" perché sottolinea una particolare falda dell'espressività filmica. E quella dell'emergenza di una immagine «mancata»o "mancante», è l'insorgenza virtuale di un ricordo "puro" al quale non si riferisce alcuna immagine concreta: lo schermo vibra - anche sonoramente, musicalmente - di una intensità che chiede, appunto, di essere colmata o, meglio, di essere condotta verso una esplicitazione che in ogni caso resta, per attimi assai significativi, incombente e non realizzata, non definita. L'immagine-ricordo di una donna, quando non è individuata in un volto e in una fisionomia precisa, lascia aperto il campo dell'immaginazione ad una potenza di forte carica drammatica e affettiva. Vero caso di donna "fantasma», in quanto tale immagine incombe ma non entra nella visualizzazione e focalizzazione dell'inquadratura, dentro lo schermo, e così il ricordo si apre alla pura dimensione della virtualità; lo spettatore resta agganciato a una promessa, a un sospiro, a un immalinconirsi del racconto, in attesa di poter verificare quando e come l'immagine della donna evocata virtualmente, amata tanto intensamente, apparirà nel suo chiaro pronunciamento audiovisivo.

Un film quasi dimenticato, Singapore (id., 1947) di John Brahm, interpretato da Ava Gardner e Fred McMurray, segue una tale falsariga concettua-

18. Cfr. Gilles Deleuze, L’image temps, Paris, 1989 (tr. it., L’immagine-tempo, Milano: UbuLibri, 1989); Gian Piero BRUNETTA, Le mille e una fonte della memoria cinematografica, in Leonardo QUARESIMA (a cura di), Il cinema e le altre arti, Venezia: Marsilio, 1996, p. 25-32; Gino FrezZA, Media di memorie, in Stefano GENSINI (a cura di), "La memoria e i segni», Quaderni del Dipartimento di Scienze della Comunicazione dell'Università di Salerno, 1, Roma: Carocci, 2000, p. 111-148. 
le-immaginaria. Il film, appartenente al genere del melò di guerra, se ne distacca per la trama affettiva fra i due personaggi principali, coinvolti in un vertiginoso intreccio memoriale. Lo spettatore segue l'avventuriero americano (Fred McMurray) da poco giunto nel bar dell'albergo di Singapore subito dopo la seconda guerra; e, per pochi secondi, il meccanismo della voce off che racconta dissolvendosi nelle immagini della storia vissuta nei giorni immediatamente precedenti l'attacco a Pearl Harbour, si avvia soltanto dopo una radicale sospensione del suo gesto e dell'espressione del volto, davanti a qualcosa che il personaggio mira con sguardo fisso, che lo spettatore può solo imprecisamente supporre, trattandosi di una scena vuota e muta (un tavolo del bar, al quale nessuno è seduto, evocante qualcosa di assente dall'immagine). Ecco la comparsa di una scaglia del tempo, di una spaccatura e di una falda di memoria che consegnano allo spettatore l'evanescente dominio di un brivido emozionale, in cui si saldano presente passato e futuro, apprensione affettiva e desiderio, nostalgia e produzione fantasmatica di una immagine intima e interiore.

Che il tema della donna fantasma sia il cardine dell'intreccio di questo film è confermato dalla operazione memoriale inversamente corrispettiva che coinvolge il personaggio della donna; ritenuta morta dopo un bombardamento dal protagonista maschile, ella — l'ammirevole Ava Gardner - in realtà è sopravvissuta ma ha del tutto perso il ricordo della sua precedente identità e dell'amore appassionato che l'aveva legata all'americano. Il nodo che si tesse fra i due amanti del passato è quindi relativo al recupero di una memoria che consenta il reincarnarsi della prima donna — quella del passato- davanti alla secon$\mathrm{da}$ — che vive, smemorata, nel presente. Questa seconda donna è una manifestazione esteriore di una immagine alla quale ella è chiamata — dal personaggio dell'americano e dagli spettatori- a conformarsi esattamente, prima o poi, nel dipanarsi della storia, rispondendo alla profondità delle sue emozioni più intime. E difatti ella subisce, nei rinnovati incontri con l'americano, l'inevitabile interferenza di emozioni apparentemente inspiegabili ed inespresse, fino a che - il film essendo un melò contenuto in un noir - non si verifica il pieno recupero di una memoria che era stata soggetta a una divaricazione fra il corpo della donna e la sua immagine/identità del passato. La donna fantasma, infine, in questo film si reincarna nella donna del presente ma al prezzo di un percorso travagliato e difficile, costantemente soggetto a incrinarsi e ad aumentare ulteriormente la distanza fra la donna e la sua immagine «ideale».

\section{Donna-idea}

L'homme qui amait les femmes, L'uomo che amava le donne, diretto nel 1977 da François Truffaut, è la storia di un impenitente adulto che ha innumerevoli relazioni con donne giovani e belle. Vi riesce grazie alla sua disarmante simpatia, alla tenera offerta che l'uomo ogni volta prospetta di sé, del proprio carattere e della sua persona, per poco o anche parecchio tempo, alle singole donne incontrate in occasioni disparate. Delle donne egli apprezza l'eleganza e la fine andatura, le gambe slanciate verso le quali resta memorabile (per gli 
appassionati seguaci del cinema di Truffaut) una sua frase: le gambe delle donne sono compassi che misurano il mondo. Tutta l'esperienza di vita del protagonista maschile di questo incomparabile film è all'insegna della centralità del femminile, della sagoma della donna da lui incessantemente inseguita, alla ricerca di qualcosa che ogni volta tenta di ritrovare. La sua vasta collezione di innamoramenti (non semplici flirts, bensì coinvolgimenti appassionati, per motivi affatto diversi) ha del vertiginoso; non semplice dongiovanni (egli non pensa né vuole consumare a proprio esclusivo vantaggio la relazione con il femminile, né è mosso da compulsione erotica o da sfrenata sensualità amorosa), Bertrand (con il viso spigoloso e simpatico di Charles Denner) evidentemente corre verso qualcosa che imprigiona la sua immaginazione ma che, prima o poi, si dilegua. E così egli ricomincia, mosso dall'impulso di star dietro a una idea che divora e consuma la sua intera attenzione.

Ma perché Bertrand ama tutte le donne? E cosa cerca inseguendo affannosamente le loro sagome slanciate e la loro differente, vivace, pungente intelligenza di vita? Tra le figure che contano nella sua vita ve ne sono tre che svolgono un ruolo chiave. La prima è Aurore, la dolce, invasiva telefonista con la quale Bertrand tesse una relazione incompleta, fatta solo di dialoghi e di un contatto fra voci. Aurore ha una voce che seduce Bertrand, lo chiama talvolta al telefono a tarda notte; una volta, Aurore chiede a Bertrand di spegnere la luce e i due si parlano al buio, cioè nella situazione di massima, disponibile, insorgenza di qualsiasi immagine. Bertrand insiste per incontrarla, ma Aurore resiste, finché gli dà un appuntamento. Bertrand è ansioso di poterla vedere, di far corrispondere alla voce suadente della donna una figura e una sagoma. Ma Aurore si sottrae; non si reca all'incontro, e Bertrand inutilmente cerca di individuarla. Aurore resta un'immagine mancata, con lei Bertrand mantiene un contatto visivo trascendente; la voce al telefono possiede un carisma che spinge l'immaginazione dell'uomo e così resterà fino alla fine. È Aurore che compie questa scelta, consapevolmente: teme di deludere l'immagine interiore, virtuale, che Bertrand ha coltivato dentro di sé, preferisce restare immagine di sogno, speranza visivamente incompleta ma emotivamente seducente. Aurore è il primo livello di esplicitazione della donna fantasma che regge la trama dei sentimenti profondi che spingono Bertrand ad amare le donne.

La seconda figura fondamentale per Bertrand è, naturalmente, la madre. Questa è rievocata dall'uomo in frammenti-ricordo che lo spettatore contempla sullo schermo in bianco/nero (a differenza della storia al presente, a colori). Bertrand rivede in memoria alcuni momenti del passato nei quali la madre è presenza sostanzialmente amata e desiderata ma sfuggente; Bertrand la sente nel ricordo con una fisionomia idealizzata, la madre concede a lui una parca attenzione che stimola in Bertrand ragazzo un amore essenzialmente rinviato, distaccato; non a caso di lei gli spettatori vedono soprattutto le gambe e porzioni del corpo. La figura della madre, intravista per sineddoche, dimostra agli spettatori l'impulso che muove Bertrand a tornare costantemente ad una seduzione originaria, a un turbamento dell'età adolescenziale che non si è mai risolto. La madre, secondo livello di donna fantasma, costituisce la matrice e il cuore 
della vicenda affettiva che spinge Bertrand. La madre è l'immagine intensa che l'uomo porta con sé ovunque ma che dilegua a ogni passo, che egli non potrà mai avere: ella è fatta di inconsistenze fuggevoli, di apparizioni che, tanto accendono le passioni e i desideri, quanto sono immateriali al pari di quella idea attorno alla quale gira l'intera esistenza di Bertrand.

La terza figura decisiva è il vecchio amore che Bertrand incontra per caso in un albergo di Parigi. La donna, Vera, è indiscutibilmente elegante e seducente (ha le dolci fattezze di una matura Leslie Caron). Con lei Bertrand ha vissuto un rapporto acutamente sentito nell'anima, ma la storia si è conclusa negativamente, senza una spiegazione sufficiente. Nell'albergo, a distanza di cinque anni dalla separazione, i due finalmente si dicono tutto. Per l'esito della loro storia, non hanno contato gli scoppi di gelosia o le banali ripicche sentimentali, se non in quanto segnalano un disagio profondissimo da parte di Bertrand, al quale va la principale responsabilità di aver condotto una straordinaria storia d'amore alla rottura improvvisa. Vera è stata l'unica donna in grado di sommare, e d'incarnare in una sola persona, l'idea virtuale della madre e la bellezza eterea della diva-donna tenacemente perseguita per un'intera vita. Ecco perché la storia con lei diviene troppo sofferta per Bertrand; dal dialogo vien fuori che è stata lei a interrompere repentinamente la storia ma dopo che Bertrand aveva dato ampi segnali di voler tornare a essere «indipendente e solitario». È a lui che tocca la responsabilità primaria di aver messo le cose in modo che il rapporto s'interrompesse. La vita con questa mirabile donna costringe Bertrand a fuoriuscire per sempre dal miraggio di una tensione immaginaria che ella realizza compiutamente, imponendogli di fare fino in fondo i conti con se stesso. Ma Bertrand non può che restare identico alla sua formazione di perpetuo innamorato, di eterno immaturo adolescente; egli non può non continuare a inseguire un modello sempre differito di seduzione e di invaghimento, di passione tanto accesa quanto sul punto di svanire come neve al sole. La conferma di questa spiegazione viene dalla breve sequenza successiva alla definitiva separazione fra $\mathrm{i}$ due; dopo la dissolvenza in nero che sigla il loro addio nell'albergo Bertrand è di nuovo a casa, di mattina; vestendosi, sfila una cravatta dall'armadio scoprendovi, sotto, tre fotografie di Vera, che lo spettatore suppone siano state lì, mirate da Bertrand ogni giorno, nel corso dei cinque anni seguiti all'interruzione del rapporto con Vera. Con l'Uomo che amava le donne, la donna fantasma incontra una tipologia essenzialmente petrarchesca di costruzione ideale secondo cui un personaggio del cinema determina la propria vita; nel film di Truffaut la donna fantasma è l'idea portante che costantemente tiene sul ciglio, reciprocamente di fronte, l'immaginazione e la realtà. L'uomo che amava le donne decifra ambedue dalla posizione rischiosa di chi vorrebbe farle corrispondere intensamente, ma è costretto o a verificare l'impossibilità di una loro coincidenza oppure a coltivare ininterrottamente la loro distanza, per poter ancora generare la visione di un oltre da inseguire, da desiderare e da acciuffare. 


\section{Conclusioni}

L'itinerario qui intrapreso sulla donna, l'immagine e il cinema - provvisorio e manchevole- può muovere qualche conclusiva osservazione, sperando di fornire a chi legge la possibilità di condividere fino in fondo l'adesione a un rapporto - quello fra la poesia di Petrarca e il cinema - che, prima d'avviare questa indagine, si pensava fosse del tutto episodico, mentre invece adesso si rivela nella cruciale valenza di un ricorrente crocevia culturale, importante e denso di sorprese.

Il tema dell'immagine della donna, in Petrarca, è indissolubilmente legato alla reazione e all'emozione che il poeta vive secondo le varie emergenze della sua figura immaginaria, della Laura non più in vita, visionaria, ideale $\mathrm{o}$ angelicata, alla quale dedica il suo percorso esistenziale e culturale. Uno dei risultati di tale trasporto emotivo sono le lagrime sparse ogni volta che gli occhi interiori del poeta la rivedono o la ripensano. Si va - in una collezione di casi molto più estesa di quella qui riportata- dal Trovommi Amor del tutto disarmato, / et aperta la via per gli occhi al core, / che di lagrime son fatti uscio et varco. (III, 9-11), al Però, dolenti, anzi che sian venutel l'ore del pianto, che son già vicine,... (XIV, 11-12), a fermo le piante sbigottito et smorto,/ et gli occhi in terra lagrimando abasso (XV, 7-8), dall'attacco di Piòvonmi amare lagrime dal viso/ con un vento angoscioso di sospiri,/ quando in voi adiven che gli occhi giri, / per cui sola dal mondo i'son diviso. (XVII, 1-4), al finale tacito vo, ché le parole mortel farian pianger la gente, et i' desio/ che le lagrime mie si spargan sole. (XVIII-1214), all'esauriente e per noi rivelatore Però con gli occhi lagrimosi e 'nfermil mio destino a vederla mi conduce; et so ben ch'i' vo dietro a quel che m'arde. (XIX, 12-14), ecc. ecc.

Nei termini della donna fantasma, la produzione dell'immagine femminile da parte di un medium tecnologico come il cinema e la sua offerta su grandi schermi che ne amplificano socialmente il consumo e la diffusione presentano un risvolto patico-affettivo non indifferente; tale immagine è strettamente correlata al fatto di provocare negli spettatori il sentimento di una incrinatura, di una esistenza in bilico, di una insorgenza complessa e difficile, di una emergenza soggetta costantemente a dissolversi. Ciò suscita reazioni non sempre programmabili o controllabili da parte degli spettatori; il desiderio di mirare, guardare, catturare — sia pure per poco - una tale immagine, è incrementato da strategie di produzione audiovisiva talvolta sofisticate ed eccelse, ma allo stesso tempo tale desiderio è sottoposto a sbocchi di rapido declino, di scomparsa o vanità del proprio oggetto, di ambivalente segnatura: fra apparente e reale, consistente e immateriale, fantastico e oggettivo, trasparente e concreto.

La donna fantasma agita il desiderio ma insieme lo nega o lo guida verso una irrisolta impasse malinconica. Il pathos da parte degli spettatori è condotto - talora sottilmente- sul punto di sciogliersi in reazione istintiva, in pianto dirompente, in lagrime che, palesemente, dichiarano al soggetto di aver mancato il desiderio di colmare una tale assenza, o di aver riconosciuta l'assenza 
stessa e quindi d'essere pervenuti a un livello di intollerabile stato affettivo. Quando poi le lagrime spettatoriali corrispondono a lagrime rappresentate di personaggi che piangono, inquadrati in primo piano, specialmente se femminili, colti in una situazione di acuto venir meno delle speranze coltivate o dei desideri frenati da accadimenti negativi, si determina un cortocircuito fra scher$\mathrm{mi}$ in sala e pathos spettatoriale che, in molti casi, genera effetti di ricorsivo diagramma, spesso metanarrativo, della comunicazione filmica. Basta citare a tal proposito un solo esempio: il viso della protagonista femminile, Nanà/Anna Karina, di Vivre sa vie, Questa è la mia vita, 1962, di Jean Luc Godard, quando, all'interno di un cinema dove sta vedendo La passion de Jeanne d'Arc di Carl Th. Dreyer, ella piange in primissimo piano davanti alle immagini della morte di Jeanne. Il destino della pulzella d'Orlèans è troppo forte perché Nanà non riconosca in lei il fantasma in cui si rispecchia la sua travagliata vita.

La sottolineatura delle lagrime per noi ha un valore ben preciso. Il pianto e le lagrime dello spettatore davanti allo schermo (che corrispondano o meno a lagrime dei personaggi o di donne viste in primo piano) costituiscono dall'epoca delle origini del cinema narrativo (dai grandi film di D.W. Griffith) uno scopo altamente specifico del pathos che il flusso d'immagini mute o sonore tesse fra schermo e pubblico. Vi sono generi filmici che hanno condotto questa densa relazione emotiva, fra immagini sullo schermo e condizione spettatoriale, a un livello di forte concentrazione, a picchi di espressività, a punte di sperimentazione: basti pensare al melò —-dalle opere di maestri classici del calibro di Douglas Sirk, Leo McCarey, dell'italiano Raffaello Matarazzo, ai film moderni di Rainer W. Fassbinder e di Tim Burton. ${ }^{19}$ Di fronte a questo strato dell'espressività filmica si pone l'urgenza di ricostruire approfonditamente i percorsi con cui si condensa in soluzioni consolidate. La via del «petrarchismo» nel cinema appare adesso una soluzione che nel tempo si è realizzata degnamente, tramite le personificazioni e la centralità della figura della donna fantasma.

Alfine, eccoci davanti alle lagrime, alle gocce umide che coprono d'improvviso gli occhi degli spettatori messi davanti a immagini che sciolgono dal controllo razionale sul proprio essere, scatenando una tempesta emotiva. La teoria critica ingenua, disinvolta o interessata, di questo effetto della visione filmica ha, in varie occasioni, peccato di supponenza cinica, se non astiosa, dicendo male delle lagrime. Invece, secondo il tracciato che origina (forse...) dal Petrarca, si deve ritenere ch'esse contengano una verità comunicativa affabile e sincera, posta in relazione organica con il senso, stringentemente individuale o potentemente collettivo, che le immagini del cinema assumono

19. Cfr. Lucilla Albano, La caverna dei giganti, Parma: Pratiche, 1992; cfr. Alberto Pezzotta (a cura di), Forme del melodramma, Roma: Bulzoni, 1992; Orio CALDIRON e Stefano Della CASA, (a cura di) Appassionatamente. Il melò nel cinema italiano, Torino: Lindau, 1999; Giovanni SPAGNOLETTI (a cura di), Lo specchio della vita. Il melodramma nel cinema contemporaneo, Torino: Lindau, 1999; Alfonso AMENDOLA, «Del tumultuoso divenire delle passioni: il melò», in Gino FreZZA (a cura di), Fino all'ultimo film. L'evoluzione dei generi nel cinema, Roma: Editori Riuniti, 2001, p. 129-161. 
davanti al loro pubblico. Al cinema si piange per motivi assai validi e mai per malintesa o vuota adesione a progetti di condizionamento (posto che teorie del condizionamento emotivo siano oggi sociologicamente, mediologicamente e culturalmente ancora proponibili, nell'epoca delle comunicazioni digitali e interattive). Ma bisogna saper far piangere: le immagini devono possedere virtù semiotiche in grado di scatenare un cortocircuito di stringente adesione con l'intimità, non poche volte segreta, che gli spettatori riconoscono sullo schermo. Il cinema classico e moderno ha saputo condurre a un egregio livello questa possibilità, senza mai sprecarla, talvolta centellinando e misurando accortamente la via d'innesto con cui le lagrime divengono un culmine espressivo, una esperienza indimenticabile.

Le lagrime sono il condensato patico che scioglie in liquido l'esserci dell'immagine interiore: questa devasta l'essere, lo scuote a fondo. L'immagine sullo schermo corrisponde a un grumo passionale riposto, al mondo privato o a quello collettivo e pubblico, spesso recondito, per altra via inconfessato, che s'accende dentro lo spettatore, collegando verticalmente presente e passato, idea e realtà, memoria e vissuto. Le lacrime spettatoriali corrispondono alle lagrime petrarchesche a fronte della vanità terrena dei mortali, del cambiamento irreversibile che il tempo cagiona togliendo amanti e bellezza e consegnando l'individuo alle risorse fantasmatiche di attesa del destino finale.

\section{Film citati}

La passion de Jeanne d'Arc, La passione di Giovanna d'Arco, 1928, regia di Carl Th. Dreyer.

Song of Songs, Il cantico dei cantici, 1933, regia di Rouben Mamoulian. Rebecca, id., 1940, regia di Alfred Hitchcock.

Phantom Lady, La donna fantasma, 1944, regia di Robert Siodmak.

Laura, Vertigine, 1944, regia di Otto Preminger.

The Uninvited, La casa sulla scogliera, 1944, regia di Lewis Allen.

The woman in the Window, La donna del ritratto, 1944, regia di Fritz Lang.

Scarlet Street, Strada Scarlatta, 1946, regia di Fritz Lang.

Singapore, id., 1947, regia di John Brahm.

Portrait of Jennie, Ritratto di Jennie, 1948, regia di William Dieterle.

That lady in ermine, La signora in ermellino, 1948, regia di Ernst Lubitsch e Otto Preminger.

One touch of Venus, Il bacio di Venere, 1948, regia di William A. Seiter.

Vertigo, La donna che visse due volte, 1958, regia di Alfred Hitchcock.

Vivre sa vie, Questa è la mia vita, 1962, regia di Jean Luc Godard.

L'homme qui amait les femmes, L'uomo che amava le donne, 1977, regia di François

Truffaut.

What lies beneath, Le verità nascoste, 2000, regia di Robert Zemeckis.

Big Fish, id., 2003, regia di Tim Burton. 